Algebra Univers. 67 (2012) 175-188

DOI 10.1007/s00012-012-0176-1

Published online February 16, 2012

(C) 2012 The Author(s)

Algebra Universalis

This article is published with open access at Springerlink.com

\title{
Intuitionistic logic and Muchnik degrees
}

\author{
Andrea Sorbi and Sebastiaan A. Terwijn
}

\begin{abstract}
We prove that there is a factor of the Muchnik lattice that captures intuitionistic propositional logic. This complements a now classic result of Skvortsova for the Medvedev lattice.
\end{abstract}

\section{Introduction}

Among the structures arising from computability theory, the lattices introduced by Medvedev and Muchnik stand out for several distinguished features and a broad range of applications. In particular, these lattices have an additional structure that makes them suitable as models of certain propositional calculi. The structure of the Medvedev lattice as a Brouwer algebra, and thus as a model for propositional logics, has been extensively studied in several papers, see e.g., [10], [15], [17], [20], [22]. Originally motivated in [10] as a formalization of Kolmogorov's calculus of problems [7], the Medvedev lattice fails to provide an exact interpretation of the intuitionistic propositional calculus IPC; however, as shown by Skvortsova [15], there are initial segments of the Medvedev lattice that model exactly IPC. On the other hand, little is known about the structure of the Muchnik lattice, and of its dual, as Brouwer algebras. The goal of this paper is to show that there are initial segments (equivalently: factors obtained dividing the lattice by principal filters) of the Muchnik lattice, in which the set of valid propositional sentences coincides with IPC. This shows that the analogue of Skvortsova's theorem also holds for the Muchnik lattice. From this, it readily follows that the propositional sentences that are valid in the Muchnik lattice are exactly the sentences of the so-called logic of the weak law of the excluded middle ([17]). Similar results (as announced, with outlined proofs, in [18]) hold for the dual of the Muchnik lattice: detailed proofs are provided in Section 5.

For all unexplained notions from computability theory, the reader is referred to Rogers [14]; our main source for Brouwer algebras and the algebraic semantics of propositional calculi is Rasiowa-Sikorski [13]. A comprehensive survey on the Medvedev and Muchnik lattices, and their mutual relationships,

Presented by C. Tsinakis.

Received December 2, 2010; accepted in final form May 25, 2011.

2010 Mathematics Subject Classification: Primary: 03D30; Secondary: 03B55, 03G10.

Key words and phrases: Muchnik lattice, Brouwer algebras, intuitionistic logic, weakly projective distributive lattice. 
can be found in [19]. Throughout the paper, we use the symbols + and $\times$ to denote the join and meet operations, respectively, in any lattice.

1.1. The Medvedev and the Muchnik lattices. Although our main object of study is the Muchnik lattice, reference to the Medvedev lattice will be sometimes useful. Therefore, we start by reviewing some basic definitions and facts concerning both lattices. Following Medvedev [10], a mass problem is a set of functions from the set of natural numbers $\omega$ to $\omega$. There are two natural ways to extend Turing reducibility to mass problems: following [10], a mass problem $\mathcal{A}$ is Medvedev-reducible to a mass problem $\mathcal{B}$ (denoted by $\mathcal{A} \leqslant_{M} \mathcal{B}$ ), if there is an oracle Turing machine by means of which every function of $\mathcal{B}$, when supplied to the machine as an oracle, computes some function of $\mathcal{A}$. (Any oracle Turing machine defines in this sense a partial mapping from $\omega^{\omega}$ to $\omega^{\omega}$, called a partial computable functional.) A different approach, which consists in dropping uniformity, leads to Muchnik reducibility, [12], denoted by $\leqslant_{w}$ : here $\mathcal{A} \leqslant_{w} \mathcal{B}$ if for every $g \in \mathcal{B}$ there is an oracle Turing machine which computes some $f \in \mathcal{A}$ when given $g$ as an oracle. This amounts to saying that $\mathcal{A} \leqslant_{w} \mathcal{B}$ if and only if for every $g \in \mathcal{B}$ there is some $f \in \mathcal{A}$ such that $f \leqslant_{T} g$. Both definitions may be viewed as attempts at formalizing Kolmogorov's idea of a calculus of problems: Kolmogorov's informal problems are now identified with mass problems; to "solve" a mass problem means to find a computable member in it; $\mathcal{A} \leqslant_{M} \mathcal{B}$ and $\mathcal{A} \leqslant_{w} \mathcal{B}$ are then formalizations of " $\mathcal{A}$ is less difficult than $\mathcal{B}$ ", as one can solve $\mathcal{A}$ given any solution to $\mathcal{B}$. In the same vein, one can introduce a formal "calculus" of mass problems, by defining $\mathcal{A}+\mathcal{B}=\{f \oplus g: f \in \mathcal{A}$ and $g \in \mathcal{B}\}$, where

$$
f \oplus g(x)= \begin{cases}f(y), & \text { if } x=2 y, \\ g(y), & \text { if } x=2 y+1 ;\end{cases}
$$

and $\mathcal{A} \times \mathcal{B}=\langle 0\rangle^{\wedge} \mathcal{A} \cup\langle 1\rangle \mathcal{B}$, where in general, for $i \in \omega$ and a given mass

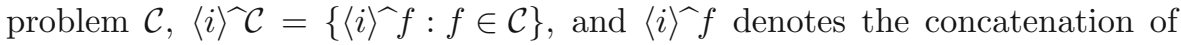
the string $\langle i\rangle$ with the function $f$. We see that $\mathcal{A}+\mathcal{B}$ has a solution if and only if both $\mathcal{A}$ and $\mathcal{B}$ have solutions; and $\mathcal{A} \times \mathcal{B}$ has a solution if and only if at least one of them has. Being preordering relations, both $\leqslant_{M}$ and $\leqslant_{w}$ give rise to degree structures: the equivalence class $\operatorname{deg}_{M}(\mathcal{A})$ of a mass problem $\mathcal{A}$, under the equivalence relation $\equiv_{M}$ generated by $\leqslant_{M}$, is called the Medvedev degree of $\mathcal{A}$; the equivalence class $\operatorname{deg}_{w}(\mathcal{A})$ of a mass problem $\mathcal{A}$, under the equivalence relation $\equiv_{w}$ generated by $\leqslant_{w}$, is called the Muchnik degree of $\mathcal{A}$. The corresponding degree structures are not only partial orders, but in fact bounded distributive lattices, with operations of join and meet (still denoted by + and $\times$ ) defined through the corresponding operations on mass problems. It is easily seen that both lattices are distributive. The lattice of Medvedev degrees is called the Medvedev lattice, denoted by $\mathfrak{M}$; the lattice of Muchnik degrees is called the Muchnik lattice, denoted by $\mathfrak{M}_{w}$. Finally, the least element 0 in 
both lattices is the degree of any mass problem containing some computable function, and the greatest element 1 is the degree of the mass problem $\emptyset$.

A Muchnik mass problem $\mathcal{A}$ is a mass problem satisfying: If $f \in \mathcal{A}$ and $f \leqslant_{T} g$, then $g \in \mathcal{A}$.

Lemma 1.1. The following hold:

(1) for every mass problem $\mathcal{A}$, there is a unique Muchnik mass problem $C(\mathcal{A})$ such that $\mathcal{A} \equiv{ }_{w} C(\mathcal{A})$;

(2) $\mathfrak{M}_{w}$ is a completely distributive complete lattice, with $\mathcal{A} \times \mathcal{B} \equiv{ }_{w} \mathcal{A} \cup \mathcal{B}$, and if $\mathcal{A}$ and $\mathcal{B}$ are Muchnik mass problems then $\mathcal{A}+\mathcal{B} \equiv_{w} \mathcal{A} \cap \mathcal{B}$.

Proof. Define $C(\mathcal{A})=\left\{f:(\exists g \in \mathcal{A})\left[g \leqslant_{T} f\right]\right\}$. The lattice $\mathfrak{M}_{w}$ is complete: if $\left\{\mathcal{A}_{i}: i \in I\right\}$ is any collection of mass problems, then the infimum and the supremum of the corresponding Muchnik degrees are given by

$$
\begin{aligned}
& \prod\left\{\operatorname{deg}_{w}\left(\mathcal{A}_{i}\right): i \in I\right\}=\operatorname{deg}_{w}\left(\bigcup\left\{\mathcal{A}_{i}: i \in I\right\}\right), \\
& \sum\left\{\operatorname{deg}_{w}\left(\mathcal{A}_{i}\right): i \in I\right\}=\operatorname{deg}_{w}\left(\bigcap\left\{C\left(\mathcal{A}_{i}\right): i \in I\right\}\right) .
\end{aligned}
$$

We will often extend the $\prod$ and $\sum$ operations to mass problems by defining:

$$
\begin{aligned}
& \prod\left\{\mathcal{A}_{i}: i \in I\right\}=\bigcup\left\{\mathcal{A}_{i}: i \in I\right\} \\
& \sum\left\{\mathcal{A}_{i}: i \in I\right\}=\bigcap\left\{C\left(\mathcal{A}_{i}\right): i \in I\right\} .
\end{aligned}
$$

Complete distributivity follows from the fact that infima and suprema are essentially given by set theoretic unions and intersections.

Both in $\mathfrak{M}$ and in $\mathfrak{M}_{w}$, a degree $\mathbf{S}$ is called a degree of solvability if it contains a singleton. The following considerations concerning degrees of solvability apply to both $\mathfrak{M}$ and $\mathfrak{M}_{w}$ : it is easy to see that the degrees of solvability form an upper semilattice, with least element, which is isomorphic to the upper semilattice, with least element, of the Turing degrees; for every degree of solvability $\mathbf{S}$, there is a unique minimal degree $>\mathbf{S}$ that is denoted by $\mathbf{S}^{\prime}$ (cf. Medvedev [10]): If $\mathbf{S}=\operatorname{deg}_{M}(\{f\})$, then $\mathbf{S}^{\prime}$ is the degree of the mass problem

$$
\{f\}^{\prime}=\left\{\langle n\rangle \widehat{\gamma}: g \mathbb{K}_{T} f \wedge \Phi_{n}(g)=f\right\},
$$

where $\left\{\Phi_{n}\right\}_{n \in \omega}$ is an effective list of all partial computable functionals; note further that for any $f$, we have $\{f\}^{\prime} \equiv_{w}\left\{g \in \omega^{\omega}: f<_{T} g\right\}$ so that in $\mathfrak{M}_{w}$ we can use this simplified version of $\{f\}^{\prime}$. In particular, $0^{\prime}=\left\{g: g>_{T} \emptyset\right\}$ is the unique minimal nonzero Muchnik degree.

\section{Brouwer algebras and intermediate propositional calculi}

We now recall the basic definitions and facts about Brouwer and Heyting algebras, and their relation with propositional logics.

Definition 2.1. A distributive lattice $L$ with least and largest elements 0 and 1 , respectively, and with operations of join and meet denoted by + and $\times$, respectively, is a Brouwer algebra if for every pair of elements $a$ and $b$, there 
is a smallest element, denoted by $a \rightarrow b$, such that $a+(a \rightarrow b) \geqslant b$. Thus, a Brouwer algebra can be viewed as an algebraic structure with three binary operations,$+ \times, \rightarrow$, together with the nullary operations 0,1 . For applications to propositional logic, it is also convenient to enrich the signature of a Brouwer algebra with a further unary operation $\neg$, given by $\neg a=a \rightarrow 1$.

Given a Brouwer algebra $L$, we can identify a propositional formula $\varphi$, having $n$ variables, with an $n$-ary polynomial $p_{\varphi}$ of $L$, in the restricted signature $\langle+, \times, \rightarrow, \neg\rangle$ : the identification makes the propositional connectives $\vee, \wedge, \rightarrow, \neg$ correspond to the operations $\times,+, \rightarrow, \neg$ of $L$, respectively. Note that $\vee$ corresponds to $\times$, not + , and dually $\wedge$ corresponds to + , not $\times$. (For polynomials in the sense of universal algebra, we refer to [3].) The polynomial $p_{\varphi}$ is a function $p_{\varphi}: L^{n} \longrightarrow L$.

Definition 2.2. Let $L$ be a Brouwer algebra. A propositional formula $\varphi$ having $n$ variables is true in $L$ if $p_{\varphi}\left(a_{0}, \ldots, a_{n-1}\right)=0$ for all $\left(a_{0}, \ldots, a_{n-1}\right) \in$ $L^{n}$. The set of all propositional formulas that are true in $L$ is denoted by $\operatorname{Th}(L)$.

The propositional formulas lying in $\operatorname{Th}(L)$ are called in [14] the identities of $L$. This is consistent with the way the term "identity" is commonly used in universal algebra: indeed, $\varphi \in \operatorname{Th}(L)$ if and only if $p_{\varphi} \approx 0$ is an identity of $L$ (with $p_{\varphi}$ and 0 regarded as terms of the type of Brouwer algebras: terminology and notations are here as in [2]).

The dual notion is studied as well.

Definition 2.3. A distributive lattice $L$ with least and largest elements 0 and 1 is a Heyting algebra if its dual $L^{\text {op }}$ is a Brouwer algebra. That is, $a \rightarrow b$ is the largest element of $L$ such that $a \times(a \rightarrow b) \leqslant b$. A propositional formula is true in the Heyting algebra $L$ (or, an identity of $L$ ) if the polynomial $p_{\varphi}^{\mathrm{op}}$, obtained from $p_{\varphi}$ by interchanging $\times$ and + , evaluates to 1 under every valuation of its variables with elements from $L$. The set of all formulas that are true in $L$ as a Heyting algebra is denoted by $\operatorname{Th}_{H}(L)$. Note that $\operatorname{Th}_{H}(L)=\operatorname{Th}\left(L^{\mathrm{op}}\right)$.

Lemma 2.4. Suppose that $L_{0}$ and $L_{1}$ are Brouwer algebras, and suppose that $F: L_{0} \longrightarrow L_{1}$ is a Brouwer homomorphism (i.e., a homomorphism of bounded lattices, which also preserves $\rightarrow$ ).

(1) If $F$ is injective, then $\operatorname{Th}\left(L_{1}\right) \subseteq \operatorname{Th}\left(L_{0}\right)$;

(2) If $F$ is surjective, then $\operatorname{Th}\left(L_{0}\right) \subseteq \operatorname{Th}\left(L_{1}\right)$.

Proof. See [13].

Given $a \leqslant b$ in a Brouwer algebra $L, L[a, b]$ denotes the interval

$$
[a, b]=\{x \in L: a \leqslant x \leqslant b\} .
$$

We abbreviate $L[0, b]$ by $L(\leqslant b)$, and we abbreviate $L[a, 1]$ by $L(\geqslant a)$.

Lemma 2.5. Suppose that $L$ is a Brouwer algebra, and let $a, b \in L$ be such that $a<b$. Then $L[a, b]$ is again a Brouwer algebra. 
Proof. Let $\rightarrow$ be the arrow operation in $L$. Then the arrow operation $\rightarrow[a, b]$ in $L[a, b]$ is given by

$$
x \rightarrow[a, b] y=a+(x \rightarrow y) .
$$

Lemma 2.6. Let $L$ be a Brouwer algebra and let $a, b, c \in L$ be such that $a<b$ and $c+a=b$. Then the mapping $f(x)=x+a$ is a Brouwer homomorphism of $L(\leqslant c)$ onto $L[a, b]$. As a consequence, $\operatorname{Th}(L(\leqslant c)) \subseteq \operatorname{Th}(L[a, b])$.

Proof. See [15, Lemma 4].

Lemma 2.7. Let $L$ be a distributive lattice, and suppose that $x \leqslant y$ and $z$ are arbitrary. Then the mapping $c \mapsto c \times z$ is a surjective lattice-theoretic homomorphism from the interval $[x, y]$ onto the interval $[x \times z, y \times z]$.

Proof. It is obvious that the mapping is a lattice-theoretic homomorphism. Surjectivity follows from the fact that if $x \times z \leqslant u \leqslant y \times z$, then $u$ is the image of $x+(u \times y)$.

\subsection{The Medvedev and the Muchnik lattices as Brouwer algebras.}

Examples of Brouwer algebras are provided by $\mathfrak{M}$ (Medvedev [10]), $\mathfrak{M}_{w}$ (Muchnik [12]), and the dual $\mathfrak{M}_{w}^{o p}$ (Sorbi [16]):

Proposition 2.8. The Muchnik lattice $\mathfrak{M}_{w}$ is both a Brouwer algebra and a Heyting algebra. The Medvedev lattice $\mathfrak{M}$ is a Brouwer algebra, but not a Heyting algebra.

Proof. $\mathfrak{M}_{w}$ is a Brouwer algebra ([12]), and a Heyting algebra ([16]) since it is a completely distributive complete lattice by Lemma 1.1. For instance, to show that $\mathfrak{M}_{w}$ is a Brouwer algebra, on mass problems take

$$
\mathcal{A} \rightarrow \mathcal{B}=\prod\left\{\mathcal{C}: \mathcal{B} \leqslant{ }_{w} \mathcal{A}+\mathcal{C}\right\}
$$

To show that $\mathfrak{M}$ is a Brouwer algebra $([10])$, on mass problems $\mathcal{A}, \mathcal{B}$, define

$$
\mathcal{A} \rightarrow \mathcal{B}=\left\{\langle n\rangle \uparrow f:(\forall g \in \mathcal{A})\left[\Phi_{n}(g \oplus f) \in \mathcal{B}\right]\right\}:
$$

it is immediate that $\mathcal{B} \leqslant_{M} \mathcal{A}+(\mathcal{A} \rightarrow \mathcal{B})$, and

$$
\mathcal{B} \leqslant_{M} \mathcal{A}+\mathcal{C} \Leftrightarrow \mathcal{A} \rightarrow \mathcal{B} \leqslant_{M} \mathcal{C} .
$$

Since Muchnik reducibility is a nonuniform version of Medvedev reducibility, we can also notice that for the $\rightarrow$ operation in the Muchnik lattice as a Brouwer algebra, one can take

$$
\mathcal{A} \rightarrow \mathcal{B}=\left\{f:(\forall g \in \mathcal{A})(\exists h \in \mathcal{B})\left[h \leqslant_{T} g \oplus f\right]\right\} .
$$

In terms of the calculus of problems, we observe that with these definitions of $\rightarrow$, for both Medvedev and Muchnik reducibility one has that $\mathcal{A} \rightarrow \mathcal{B}$ is a mass problem such that any solution to it, together with any solution to $\mathcal{A}$, gives a solution to $\mathcal{B}$. Thus, this implements the modus ponens proof rule.

That $\mathfrak{M}$ is not a Heyting algebra was proved in Sorbi [16, Theorem 5.4]. 
For either $\mathfrak{M}$ or $\mathfrak{M}_{w}$, Definition 2.2 amounts to saying that a propositional sentence is valid if and only if every substitution of mass problems to the propositional variables in the sentence yields a solvable problem. Let IPC denote the intuitionistic propositional calculus (see [13] for a suitable definition of axioms and rules of inference), and let Jan be the intermediate propositional logic obtained by adding to IPC the so called weak law of the excluded middle, i.e., the axiom scheme $\neg \alpha \vee \neg \neg \alpha$, where $\alpha$ is any propositional sentence. It is known (Medvedev [11], Jankov [5], Sorbi [17]) that $\operatorname{Th}(\mathfrak{M})=$ Jan. Also, $\operatorname{Th}\left(\mathfrak{M}_{w}\right)=$ Jan (announced in [17]).

By lattice theory, if $L$ is a Brouwer algebra and $b \in L$, then the Brouwer algebra $L(\leqslant b)$ is lattice isomorphic to the quotient lattice obtained by dividing $L$ modulo the principal filter generated by $b$; likewise, $L(\geqslant a)$ is isomorphic to the quotient lattice obtained by dividing $L$ modulo the principal ideal generated by $a$. The difference between these two quotients, see, e.g., [13], is that lattice-theoretic congruences given by ideals are also congruences of Brouwer algebras, and thus there is a surjective Brouwer homomorphism from $L$ into $L(\geqslant a)$, giving $\operatorname{Th}(L) \subseteq \operatorname{Th}(L(\geqslant a))$ by Lemma 2.4. In order to find exact interpretations of IPC in terms of mass problems, one should then turn attention to initial segments of the Medvedev lattice, i.e., to Brouwer algebras of the form $\mathfrak{M}(\leqslant \mathbf{A})$, where $\mathbf{A}$ is a nonzero Medvedev degree.

Theorem 2.9. (Skvortsova [15]) There exists A such that $\operatorname{Th}(\mathfrak{M}(\leqslant \mathbf{A}))=$ IPC.

It is still an open problem (raised by Skvortsova [15, p.134]) whether there is a Medvedev degree $\mathbf{A}$ that is the infimum of finitely many Muchnik degrees (i.e., Medvedev degrees containing Muchnik mass problems) such that $\operatorname{Th}(\mathfrak{M}(\leqslant \mathbf{A}))$ coincides with IPC. The paper [20] is dedicated to initial segments of the Medvedev lattice and their theories as intermediate propositional logics. Note that it does not make sense to ask whether Theorem 2.9 holds for the dual of $\mathfrak{M}$, since $\mathfrak{M}$ is not a Heyting algebra by [16]. In Section 4, we show that Theorem 2.9 also holds for $\mathfrak{M}_{w}$, and in Section 5 , we show that it holds for the dual of $\mathfrak{M}_{w}$.

\section{Capturing IPC with Brouwer and Heyting algebras}

Consider the following classic result about IPC due to McKinsey and Tarski, that provides an algebraic semantics for IPC using Brouwer algebras. (The result also follows from the results in Jaśkowski [6]).

Theorem 3.1. (Jaśkowski [6], McKinsey and Tarski [9])

$$
\begin{aligned}
\text { IPC } & =\bigcap\{\operatorname{Th}(B): B \text { a finite Brouwer algebra }\} \\
& =\bigcap\left\{\operatorname{Th}_{H}(H): H \text { a finite Heyting algebra }\right\} .
\end{aligned}
$$


We wish to narrow down the family of Brouwer algebras and Heyting algebras needed for this result, in order to suit our needs in the next section. The result we will need later is formulated below as Corollary 3.11.

For a given lattice $L$, let $J(L)$ denote the partial order of nonzero joinirreducible elements of $L$. Recall the well-known duality between finite posets and finite distributive lattices. Obviously, for every finite distributive lattice $L, J(L)$ is a poset, and conversely, for every finite poset $P$, we obtain a finite distributive lattice $H(P)$ by considering the downwards closed subsets of $P([4$, Theorem II.1.9]). These operations are inverses of each other, as $H(J(L)) \simeq L$ (as lattices), and $J(H(P)) \simeq P$ (as posets).

The following is a useful notion from the theory of categories. An equational category is a category whose objects form a variety of algebras, and whose morphisms are just the homomorphisms.

Definition 3.2. An object of an equational category $L$ is weakly projective if for every onto morphism $f: L_{0} \rightarrow L_{1}$ and every morphism $g: L \rightarrow L_{1}$, there exists a morphism $h: L \rightarrow L_{0}$ such that $g=f \circ h$. (If one changes "onto" in this definition to "epi" then one obtains the definition of projective object.)

Lemma 3.3. A distributive lattice $L$ is weakly projective if and only if its dual $L^{\text {op }} i s$.

Proof. It can be shown, see, e.g., [1, Theorem 1.14], that in a nontrivial equational category, an object is weakly projective if and only if it is a retract of a free algebra. (Recall that $A$ is a retract of $B$, if there are morphisms $f: A \rightarrow B, g: B \rightarrow A$ such that $g \circ f=1_{A}$.) If $L$ is weakly projective, and $L$ is a retract of a free distributive lattice $F$, then $L^{\text {op }}$ is a retract of $F^{\text {op }}$ which is still free.

When considering the category of distributive lattices, the following useful characterization of the finite weakly projective objects is available:

Theorem 3.4. [1, Corollary V.10.9] A finite distributive lattice $L$ is weakly projective if and only if whenever $a$ and $b$ are join-irreducible in $L$ also $a \times b$ is join-irreducible.

The following property from [23] gives an alternative characterization of finite weakly projective distributive lattices:

Definition 3.5. A finite distributive lattice $L$ is double diamond-like (dd-like, for short) if in the poset $J(L)$ there are two incomparable elements with at least two minimal upper bounds.

Proposition 3.6. A finite distributive lattice $L$ is weakly projective if and only if it is not dd-like.

Proof. When $L$ is weakly projective then every pair $a, b$ of join-irreducible elements has a greatest lower bound $a \times b$ that is join-irreducible, and hence 
$a \times b$ is also the greatest lower bound of $a$ and $b$ in the poset $J(L) \cup\{0\}$. Hence, $L$ is not dd-like.

Conversely, if $L$ is not weakly projective, then there are $a, b \in J(L)$ such that $a \times b$ is join-reducible. Without loss of generality, we can assume that $a$ and $b$ are minimal in the sense that there are no elements of $J(L)$ in between $a$ and $a \times b$, and also no elements of $J(L)$ in between $b$ and $a \times b$. Since any element in a finite distributive lattice can be written as a finite join of joinirreducible elements, there is a finite set $X \subseteq J(L)$ such that $a \times b=\sum X$. Since $a \times b$ itself is join-reducible, there are at least two maximal elements $x, y \in X$. Then both $a$ and $b$ are minimal upper bounds of $x$ and $y$ in $J(L)$, hence $L$ is dd-like.

We now undertake the task of characterizing IPC by suitably restricted families of Heyting algebras and Brouwer algebras. We can in fact start from a family that was already used by Jaśkowski, by observing that it has certain additional properties. The result we will need later is formulated below as Corollary 3.9.

Lemma 3.7. If $A$ and $B$ are finite distributive lattices that are not dd-like, then also their Cartesian product $A \times B$ is not dd-like.

Proof. We need in fact that only one of $A$ and $B$ is not dd-like. Suppose that $A$ is not dd-like. Note that $(a, b) \in A \times B$ is join-irreducible if and only if $a \in J(A)$ and $b \in J(B)$. Suppose that $A \times B$ is not dd-like, say $J(A \times B)$ contains the following configuration:

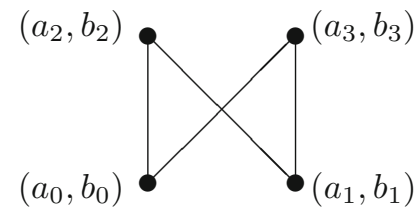

Here the pairs $\left(a_{2}, b_{2}\right)$ and $\left(a_{3}, b_{3}\right)$ are minimal upper bounds for $\left(a_{0}, b_{0}\right)$ and $\left(a_{1}, b_{1}\right)$ in $J(A \times B)$. Then in $J(A)$, the elements $a_{2}$ and $a_{3}$ are upper bounds for $a_{0}$ and $a_{1}$. Since by assumption $A$ is not dd-like, not both of $a_{2}$ and $a_{3}$ are minimal upper bounds. Say $a_{2}$ is not minimal, and that $a_{0}, a_{1} \leqslant a<a_{2}$ in $J(A)$. Replacing $\left(a_{2}, b_{2}\right)$ by $\left(a, b_{2}\right)$, we see that $\left(a_{2}, b_{2}\right)$ was not a minimal upper bound of $\left(a_{0}, b_{0}\right)$ and $\left(a_{1}, b_{1}\right)$, contrary to assumption.

We use the following result of Jaśkowski [6], (cited in Szatkowski [21, p41]). Given two Heyting algebras $A$ and $B$, let $A+B$ be the algebra obtained by stacking $B$ on top of $A$, identifying $0_{B}$ with $1_{A}$. (This notion of sum is from Troelstra [24].) Given $A$ and $B$, the Cartesian product $A \times B$ is again a Heyting algebra. Let $A^{n}$ denote the $n$-fold product of $A$.

Inductively define the following sequence of Heyting algebras. Let $I_{1}$ be the two-element Boolean algebra, and let $I_{n+1}=I_{n}^{n}+I_{1}$.

The following theorem characterizes IPC in terms of Heyting algebras: 
Theorem 3.8. (Jaśkowski [6]) IPC $=\bigcap_{n} \operatorname{Th}_{H}\left(I_{n}\right)$.

Corollary 3.9. There is a collection $\left\{H_{n}\right\}_{n \in \omega}$ of finite Heyting algebras such that

$$
\mathrm{IPC}=\bigcap_{n} \mathrm{Th}_{H}\left(H_{n}\right),
$$

and such that for every $n, H_{n}$ is weakly projective.

Proof. Note that the lattices $I_{n}$ defined above are all distributive lattices, and because they are finite, they are automatically Heyting algebras. We claim that every $I_{n}$ is not dd-like. This is clearly true for $n=1$. Suppose that $I_{n}$ is not dd-like. Then by Lemma 3.7, also $I_{n}^{n}$ is not dd-like. It follows immediately that $I_{n+1}=I_{n}^{n}+I_{1}$ is also not dd-like. Hence, all $I_{n}$ are finite Heyting algebras that are not dd-like, and hence we can simply take $H_{n}=I_{n}$.

Corollary 3.10. There is a collection $\left\{B_{n}\right\}_{n \in \omega}$ of finite Brouwer algebras such that

$$
\mathrm{IPC}=\bigcap_{n} \mathrm{Th}\left(B_{n}\right),
$$

and such that for every $n, B_{n}$ is weakly projective.

Proof. Consider any propositional formula $\varphi \notin$ IPC. Then by Corollary 3.9, there exists a weakly projective finite distributive lattice $H_{n}$ and an evaluation of $p_{\varphi}^{\text {op }}$ for which $p_{\varphi}^{\text {op }} \neq 1$, and thus, for this evaluation in $H_{n}^{\text {op }}, p_{\varphi} \neq 0$, showing that $\varphi \notin \operatorname{Th}\left(H_{n}^{\mathrm{op}}\right)$. It remains to show that $B_{n}=H_{n}^{\text {op }}$ is weakly projective: this follows from Lemma 3.3.

An easy way to obtain Corollary 3.10 would be to show that every finite distributive lattice is the image of a weakly projective finite distributive lattice under a Brouwer-homomorphism. (Corollary 3.10 would then follow immediately from Theorem 3.1 and Lemma 2.4 (2).) However, this is not true: One can prove that every finite distributive lattice is the image of a weakly projective finite distributive lattice under a lattice-homomorphism, but in general not under a Brouwer-homomorphism.

Summarizing, we have:

\section{Corollary 3.11.}

$$
\begin{aligned}
\mathrm{IPC} & =\bigcap\{\operatorname{Th}(B): B \text { a finite weakly projective Brouwer algebra }\} \\
& =\bigcap\left\{\operatorname{Th}_{H}(H): H \text { a finite weakly projective Heyting algebra }\right\} .
\end{aligned}
$$

\section{A factor of the Muchnik lattice that captures IPC}

In this section, we prove that there is a factor of $\mathfrak{M}_{w}$, obtained by dividing $\mathfrak{M}_{w}$ by a principal filter, that has IPC as its theory. Hence, we see that the analogue of Skvortsova's result (Theorem 2.9) holds for $\mathfrak{M}_{w}$. We will be very liberal with notation, frequently confusing Muchnik degrees with their representatives. 
The property of dd-like lattices (Definition 3.5) was used to characterize the lattices that are isomorphic to an interval of $\mathfrak{M}_{w}$ :

Theorem 4.1. (Terwijn [23]) For any finite distributive lattice L, the following are equivalent:

(i) $L$ is isomorphic to an interval in $\mathfrak{M}_{w}$;

(ii) $L$ is not double diamond-like;

(iii) L does not have a double diamond-like lattice as a subinterval.

Let $\left\{B_{n}\right\}_{n \in \omega}$ be the family of Brouwer algebras from Corollary 3.10. Since $B_{n}$ is not dd-like by Proposition 3.6, by Theorem 4.1 there are sets $\mathcal{X}_{n}$ and $\mathcal{Y}_{n}$ such that the interval $\left[\mathcal{X}_{n}, \mathcal{Y}_{n}\right]$ in $\mathfrak{M}_{w}$ is isomorphic to $B_{n}$ for every $n$. This is an isomorphism of finite distributive lattices; hence, it is automatically an isomorphism of Brouwer algebras.

It is useful to remind the reader of some of the details of the construction in [23]. Let $J_{n}=J\left(B_{n}\right)$ be the set of the nonzero join-irreducible elements of $B_{n}$; since $B_{n}$ is not dd-like, $J_{n}$ is an initial segment of an upper semilattice. Embed $J_{n}$ as an interval of the Turing degrees (this can be done by a classical result of Lachlan and Lebeuf [8], stating that for every Turing degree a, every countable upper semilattice with least element is isomorphic to an interval of the Turing degrees with bottom a). For every Turing degree in the range of this embedding, choose a representative, as a function $f \in \omega^{\omega}$; for convenience, let us identify $J_{n}$ with the set of these chosen representatives. For every $A \subseteq J_{n}$, let $\hat{A}$ denote the elements of $A$ that are $\leqslant_{T}$-maximal, i.e., maximal with respect to Turing reducibility.

Inspection of the proof of Theorem 3.11 in [23] shows that there is a set $\mathcal{Z}_{n}$ such that

$$
\begin{aligned}
& \mathcal{X}_{n}=\mathcal{Z}_{n} \times J_{n}, \\
& \mathcal{Y}_{n}=\mathcal{Z}_{n} \times \prod\left\{\{f\}^{\prime}: f \in \hat{J}_{n}\right\} .
\end{aligned}
$$

Furthermore, we have that $\mathcal{Z}_{n}=\bigcup_{f \in J_{n}} \mathcal{Z}_{n}^{f}$, where

$$
\mathcal{Z}_{n}^{f}=\left\{g \in\{f\}^{\prime}:\left.g\right|_{T} h \text { for all covers } h \text { of } f \text { in } J_{n}\right\} .
$$

The sets $J_{n}$ come from embedding results into the Turing degrees, and we have rather great freedom in picking them. In particular, we may pick them such that they satisfy that for every $n \neq m$,

$$
\left(\forall f \in J_{n}\right)\left[\{f\} \ngtr_{w} \mathcal{Z}_{m}\right],
$$

and

$$
\left.\begin{array}{c}
f \in \hat{J}_{m} \\
g \in \hat{J}_{n} \\
h \in J_{n}
\end{array}\right\} \Longrightarrow f \oplus h>_{T} g .
$$

To obtain this, it is enough to embed as an interval of the Turing degrees the upper semilattice $J$ defined as follows: First, let $U=\bigcup_{n}\{n\} \times J_{n}$ (where, again, $\left.J_{n}=J\left(B_{n}\right)\right)$ and in $U$ define $(n, x) \leqslant(m, y)$ if and only if $n=m$ and, 
in $J_{n}, x \leqslant y$; finally define $J$ by adding a least element and a greatest element to $U$. Clearly, $J$ is a countable upper semilattice with least element, and thus can be embedded as an interval of the Turing degrees: under this embedding, each $J_{n}$ is embedded as an interval of the Turing degrees, with the desired properties.

Define

$$
\begin{aligned}
\mathcal{Z} & =\bigcup_{n \in \omega} \mathcal{Z}_{n}, \\
\hat{\mathcal{X}}_{n} & =\mathcal{Z} \times J_{n} \equiv_{w} \mathcal{X}_{n} \times \mathcal{Z} \\
\hat{\mathcal{Y}}_{n} & =\mathcal{Z} \times \prod\left\{\{f\}^{\prime}: f \in \hat{J}_{n}\right\} \equiv_{w} \mathcal{Y}_{n} \times \mathcal{Z} .
\end{aligned}
$$

Lemma 4.2. The interval $\left[\mathcal{X}_{n}, \mathcal{Y}_{n}\right]$ is isomorphic to the interval $\left[\hat{\mathcal{X}}_{n}, \hat{\mathcal{Y}}_{n}\right]$.

Proof. Define a mapping from $\left[\mathcal{X}_{n}, \mathcal{Y}_{n}\right]$ to $\left[\mathcal{X}_{n} \times \mathcal{Z}, \mathcal{Y}_{n} \times \mathcal{Z}\right]$ by $\mathcal{C} \mapsto \mathcal{C} \times \mathcal{Z}$. By Lemma 2.7, the mapping is a surjective lattice-theoretic homomorphism. We check that it is also injective: Suppose that $\mathcal{C}_{0}, \mathcal{C}_{1} \in\left[\mathcal{X}_{n}, \mathcal{Y}_{n}\right]$ and that $\mathcal{C}_{0} \times \mathcal{Z} \equiv{ }_{w} \mathcal{C}_{1} \times \mathcal{Z}$. We claim that $\mathcal{C}_{0} \geqslant{ }_{w} \mathcal{C}_{1} \times \mathcal{Z}_{n}$ : Suppose that $g \in \mathcal{C}_{0}$. Then $\{g\} \geqslant_{w} \mathcal{X}_{n}=\mathcal{Z}_{n} \times J_{n}$. If $\{g\} \geqslant{ }_{w} \mathcal{Z}_{n}$ then clearly it can be mapped to $\mathcal{C}_{1} \times \mathcal{Z}_{n}$. If $\{g\} \ngtr_{w} \mathcal{Z}_{n}$, then we have $\{g\} \geqslant_{w} J_{n}$, and it follows from (2) and the fact that the Turing degrees of functions in $J_{n}$ form an initial segment of the Turing degrees, that $g \equiv_{T} k$ for some $k \in J_{n}$. (To see this, suppose that $\{g\} \ngtr_{w} \mathcal{Z}_{n}$ and $\{g\} \geqslant_{w} J_{n}$, and let $f \in J_{n}$ be $\leqslant_{T}$-maximal such that $f \leqslant_{T} g$. If $f \equiv_{T} g$, then the claim is true, so suppose that $f<_{T} g$. Then $g \in\{f\}^{\prime}$. There is a cover $h \in J_{n}$ of $f$ such that $g \chi_{T} h$, since otherwise $g \in \mathcal{Z}_{n}^{f}$, and hence $g \in \mathcal{Z}_{n}$, contrary to assumption. If $g \leqslant_{T} h$, then $g \equiv_{T} k$ for some $k \in J_{n}$ since the Turing degrees of the elements of $J_{n}$ are an initial segment. If $h \leqslant_{T} g$, then $g \equiv_{T} h$ since we chose $f \in J_{n}$ maximal. Hence, $g$ has the same Turing degree of some function in $J_{n}$.) But in this case, it follows from (3) and the assumption $\mathcal{C}_{0} \geqslant{ }_{w} \mathcal{C}_{1} \times \mathcal{Z}$ that $\{g\} \geqslant_{w} \mathcal{C}_{1} \times \mathcal{Z}_{n}$. Hence, $\mathcal{C}_{0} \geqslant_{w} \mathcal{C}_{1} \times \mathcal{Z}_{n} \equiv_{w} \mathcal{C}_{1}$ (note that $\mathcal{Z}_{n} \geqslant{ }_{w} \mathcal{C}_{1}$ since $\mathcal{Y}_{n} \geqslant_{w} \mathcal{C}_{1}$ ), and symmetrically we have that $\mathcal{C}_{1} \geqslant{ }_{w} \mathcal{C}_{0}$, hence $\mathcal{C}_{0} \equiv{ }_{w} \mathcal{C}_{1}$

Now let $\hat{\mathcal{Y}}=\bigcup_{n \in \omega} \hat{\mathcal{Y}}_{n}$.

Lemma 4.3. $\hat{\mathcal{Y}}+\hat{\mathcal{X}}_{n} \equiv_{w} \hat{\mathcal{Y}}_{n}$ for every $n$.

Proof. The direction $\leqslant w$ is immediate from $\hat{\mathcal{Y}} \leqslant w \hat{\mathcal{Y}}_{n}$ and $\hat{\mathcal{X}}_{n} \leqslant{ }_{w} \hat{\mathcal{Y}}_{n}$. For the other direction, suppose that $g \in \hat{\mathcal{Y}}$ and $h \in \hat{\mathcal{X}}_{n}$. We have to show that $g \oplus h$ computes some function in $\hat{\mathcal{Y}}_{n}$. Suppose that $g \in \hat{\mathcal{Y}}_{m}$. If $n=m$, then we are

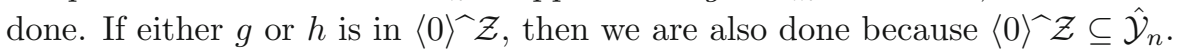

In the remaining case, we have $n \neq m, h \in\langle 1\rangle^{\wedge} J_{n}$, and $g \in\{f\}^{\prime}$ for some $f \in \hat{J}_{m}$. Let $l$ be any element of $\hat{J}_{n}$. Then by (4), we have $f \oplus h>_{T} l$, hence $g \oplus h \geqslant_{T} f \oplus h \in\{l\}^{\prime} \geqslant_{w} \hat{\mathcal{Y}}_{n}$.

Theorem 4.4. There exists a mass problem $\hat{\mathcal{Y}}$ such that

$$
\operatorname{Th}\left(\mathfrak{M}_{w}\left(\leqslant_{w} \hat{\mathcal{Y}}\right)\right)=\text { IPC. }
$$


Proof. Let $\hat{\mathcal{X}}_{n}, \hat{\mathcal{Y}}_{n}$, and $\hat{\mathcal{Y}}$ be as above. Since by Lemma 4.3 , we have $\hat{\mathcal{Y}}+\hat{\mathcal{X}}_{n} \equiv_{w}$ $\hat{\mathcal{Y}}_{n}$ for every $n$, so by Lemma 2.6 , we have that

$$
\operatorname{Th}\left(\mathfrak{M}_{w}(\leqslant w \hat{\mathcal{Y}})\right) \subseteq \bigcap_{n} \operatorname{Th}\left(\left[\hat{\mathcal{X}}_{n}, \hat{\mathcal{Y}}_{n}\right]\right)=\bigcap_{n} \operatorname{Th}\left(B_{n}\right)=\mathrm{IPC} .
$$

The equality $\operatorname{Th}\left(\mathfrak{M}_{w}\left(\leqslant_{w} \hat{\mathcal{Y}}\right)\right)=\operatorname{IPC}$ follows since IPC $\subseteq \operatorname{Th}\left(\mathfrak{M}_{w}\left(\leqslant{ }_{w} \hat{\mathcal{Y}}\right)\right)$ holds for any $\hat{\mathcal{Y}}$.

\section{5. $\mathfrak{M}_{w}$ as a Heyting algebra}

For the dual of $\mathfrak{M}_{w}$, we have a similar result, but easier to prove and in fact stronger: the result, and its consequences, listed below, were already noticed in Sorbi [18], with only a sketched proof.

Let $\left\{H_{n}\right\}_{n \in \omega}$ be the family of Heyting algebras from Corollary 3.9. The following lemma is a reformulation of a result in [23], using Proposition 3.6. The right-to left implication appeared also in [18].

Lemma 5.1. A finite distributive lattice is isomorphic to an initial segment of the Muchnik lattice if and only if it is weakly projective, and 0 is meetirreducible.

Theorem 5.2. IPC $=\mathrm{Th}_{H}\left(\mathfrak{M}_{w}\left(\geqslant 0^{\prime}\right)\right)$.

Proof. For every weakly projective finite distributive lattice $H$, define $H^{+}=$ $H+I_{1}$ (using the notation of Section 3.) Notice that $H$ is isomorphic to a factor of $H^{+}$, obtained by dividing by the principal filter generated by $1_{H}$, that is, the image of the top element of $H$ into $H^{+}$. Since filters provide congruences of Heyting algebras, we have by Lemma 2.4 (or rather, its dual version for Heyting algebras) that $\mathrm{Th}_{H}\left(H^{+}\right) \subseteq \mathrm{Th}_{H}(H)$. It follows that

$\mathrm{IPC}=\bigcap\left\{\mathrm{Th}_{H}(H): H\right.$ finite, weakly projective, with join-irreducible 1$\}$.

Suppose now that $H$ is a finite, weakly projective distributive lattice, with join-irreducible 1: let $H^{-}$be such that $H=\left(H^{-}\right)^{+}$. Embed $I_{1}+H^{-}$as an initial segment of $\mathfrak{M}_{w}$, which is possible by Lemma 5.1. Let $F$ be the embedding, which is also a Heyting algebra embedding since the range of $F$ is an initial segment. Then the mapping

$$
G(x)= \begin{cases}F(x), & \text { if } x \in H^{-}, \\ \mathbf{1}_{\mathfrak{M}_{w}}, & \text { if } x=1_{H},\end{cases}
$$

is a Heyting embedding of $H$ into $\left.\mathfrak{M}_{w}\left(\geqslant_{w} 0^{\prime}\right)\right)$. Thus, IPC $=\operatorname{Th}_{H}\left(\mathfrak{M}_{w}\left(\geqslant_{w} 0^{\prime}\right)\right)$ by Lemma 2.4 .

A proof of the following result was already outlined in Sorbi [18].

Theorem 5.3. $\operatorname{Th}_{H}\left(\mathfrak{M}_{w}\right)=\operatorname{Th}\left(\mathfrak{M}_{w}\right)=\operatorname{Jan}$. 
Proof. Let us show that $\operatorname{Th}_{H}\left(\mathfrak{M}_{w}\right)=$ Jan. For every Heyting algebra $H$, let $H_{+}=I_{1}+H$. Let us say that a propositional formula is positive if it does not contain the connective $\neg$, and for every Heyting algebra $H$, let $\operatorname{Th}_{H}^{\text {pos }}(H)=$ $\left\{\varphi \in \mathrm{Th}_{H}(H): \varphi\right.$ positive $\}$. We claim that $\operatorname{Th}_{H}^{\text {pos }}\left(H_{+}\right) \subseteq \operatorname{Th}_{H}^{\text {pos }}(H)$. Namely, one can show by induction on the complexity of a positive $\varphi$ that for every $\bar{x} \in H^{n}, p_{\varphi}^{H}(\bar{x})=p_{\varphi}^{H_{+}}(\bar{x})$. (Here, $\varphi$ has, say, $n$ variables, and $p_{\varphi}^{H}$ and $p_{\varphi}^{H_{+}}$ denote the mappings $\varphi^{\mathrm{op}}: H^{n} \rightarrow H$ and $\varphi^{\mathrm{op}}:\left(H_{+}\right)^{n} \rightarrow H_{+}$, respectively, as in Definition 2.3.) Notice also that for every Heyting algebra $H$ and any propositional formula $\alpha$, we have that $\neg \alpha \vee \neg \neg \alpha \in \operatorname{Th}_{H}\left(H_{+}\right)$(since the least element of $H_{+}$is meet-irreducible), i.e., Jan $\subseteq \operatorname{Th}_{H}\left(H_{+}\right)$. Let $H=\mathfrak{M}_{w}(\geqslant$ $\left.0^{\prime}\right)$ ), so that $H_{+}=\mathfrak{M}_{w}$. By Theorem 5.2, we have IPC $=\operatorname{Th}_{H}(H)$; hence, $\mathrm{IPC}^{\text {pos }}=\operatorname{Th}_{H}^{\text {pos }}\left(H_{+}\right)$and $\neg \alpha \vee \neg \neg \alpha \in \mathrm{Th}_{H}\left(H_{+}\right)$. Therefore, one can apply a classic result due to Jankov [5], stating that Jan is the $\subseteq$-largest intermediate propositional logic $I$ such that $\mathrm{IPC}^{\text {pos }}=I^{\text {pos }}$ and $\neg \alpha \vee \neg \neg \alpha \in I$. Thus, we also obtain the converse inclusion $\operatorname{Th}_{H}\left(H_{+}\right) \subseteq$ Jan.

The proof that $\operatorname{Th}\left(\mathfrak{M}_{w}\right)=$ Jan goes like this: let $B=\mathfrak{M}_{w}(\leqslant w \hat{\mathcal{Y}})$, with $\hat{\mathcal{Y}}$ as in Theorem 4.4. Dualizing the arguments which have been used above, we obtain $\operatorname{Th}^{\text {pos }}\left(B^{+}\right) \subseteq \operatorname{Th}^{\text {pos }}(B)$, but then again by Jankov $[5], \operatorname{Th}\left(B^{+}\right)=$Jan, and since $B^{+}$is Brouwer embeddable into $\mathfrak{M}_{w}$ (use $G: B^{+} \longrightarrow \mathfrak{M}_{w}$ which extends the embedding of $B$ into $\mathfrak{M}_{w}$, by $\left.G\left(1_{B^{+}}\right)=\mathbf{1}_{\mathfrak{M}_{w}}\right)$, we finally get that $\operatorname{Th}\left(\mathfrak{M}_{w}\right) \subseteq$ Jan (by Lemma 2.4), and thus $\operatorname{Th}\left(\mathfrak{M}_{w}\right)=$ Jan since $\neg \alpha \vee \neg \neg \alpha \in$ $\operatorname{Th}\left(\mathfrak{M}_{w}\right)$.

\section{REFERENCES}

[1] Balbes, R., Dwinger, P.: Distributive Lattices, University of Missouri Press, Columbia (1974)

[2] Burris, S., Sankappanavar, H. P.: A Course in Universal Algebra, Graduate Texts in Mathematics. Springer Verlag, New York, Heidelberg, Berlin (1981)

[3] Grätzer, G.: Universal Algebra, 2nd edn. Springer-Verlag, New York (1979)

[4] Grätzer, G.: General lattice theory, 2nd edn. Birkhäuser, Basel (2003)

[5] Jankov, V.A.: The calculus of the weak law of excluded middle. Izv. Math. 2, 997-1004 (1968)

[6] Jaśkowski, S.: Recherches sur le système de la logique intuitioniste. In: Actes du Congrès International de Philosophie Scientifique VI, Philosophie des Mathématiques, Actualités Scientifiques et Industrielles, vol. 393, pp. 8-61, Hermann, Paris (1936)

[7] Kolmogorov, A.: Zur Deutung der intuitionistischen Logik, Math. Z. 35, 58-65 (1932)

[8] Lachlan, A.H., Lebeuf, R.: Countable initial segments of the degrees of unsolvability, J. Symbolic Logic 41, 289-300 (1976)

[9] McKinsey, J.C.C., Tarski, A.: Some theorems about the sentential calculi of Lewis and Heyting. J. Symbolic Logic 13, 1-15 (1948)

[10] Medvedev, Yu.T.: Degrees of difficulty of the mass problems. Dokl. Akad. Nauk 104, 501-504 (1955)

[11] Medvedev, Yu.T.: Finite problems. Dokl. Akad. Nauk (NS) 142, 1015-1018 (1962)

[12] Muchnik, A.A.: On strong and weak reducibility of algorithmic problems. Sibirsk. Mat. Zh. 4, 1328-1341, (1963) (Russian)

[13] Rasiowa, H., Sikorski, R.: The Mathematics of Metamathematics. Panstowe Wydawnictwo Naukowe, Warszawa (1963)

[14] Rogers H., Jr.: Theory of Recursive Functions and Effective Computability. McGraw-Hill, New York (1967) 
[15] Skvortsova, E.Z.: Faithful interpretation of the intuitionistic propositional calculus by an initial segment of the Medvedev lattice. Sibirsk. Mat. Zh. 29, 171-178 (1988) (Russian)

[16] Sorbi, A.: Some remarks on the algebraic structure of the Medvedev lattice. J. Symbolic Logic 55, 831-853 (1990)

[17] Sorbi, A.: Embedding Brouwer algebras in the Medvedev lattice. Notre Dame J. Formal Logic 32, 266-275 (1991)

[18] Sorbi, A.: Some quotient lattices of the Medvedev lattice. Z. Math. Logik Grundlag. Math. 37, 167-182 (1991)

[19] Sorbi, A.: The Medvedev lattice of degrees of difficulty. In: Cooper, S.B., Slaman, T.A., Wainer, S.S. (eds.) Computability, Enumerability, Unsolvability - Directions in Recursion theory, London Mathematical Society Lecture Notes Series, pp. 289-312. Cambridge University Press, New York (1996)

[20] Sorbi, A., Terwijn, S.A.: Intermediate logics and factors of the Medvedev lattice. Ann. Pure Appl. Logic 155, 69-86 (2008)

[21] Szatkowski, M.: On fragments of Medvedev's logic. Studia Logica 40, 39-54 (1981)

[22] Terwijn, S.A.: Constructive logic and the Medvedev lattice. Notre Dame J. Formal Logic 47, 73-82 (2006)

[23] Terwijn, S.A.: The finite intervals of the Muchnik lattice. Trans. Amer. Math. Soc. (in press)

[24] Troelstra, A.S.: On intermediate propositional logics. Indag. Math. 27, 141-152 (1965)

\section{ANDREA SORBI}

University of Siena, Dipartimento di Scienze Matematiche ed Informatiche "Roberto Magari", Pian dei Mantellini 44, 53100 Siena, Italy.

e-mail: sorbi@unisi.it

\section{Sebastiann A. Terwijn}

Radboud University Nijmegen, Department of Mathematics, P.O. Box 9010, 6500 GL Nijmegen, the Netherlands.

e-mail: terwijn@math.ru.nl

Open Access This article is distributed under the terms of the Creative Commons Attribution License which permits any use, distribution, and reproduction in any medium, provided the original author(s) and source are credited. 